\title{
PREVALENCIA DE MALOCLUSIONES EN ESCOLARES DE 12 AÑOS DE LA PARROQUIA MACHÁNGARA EN LA CIUDAD DE CUENCA, 2016
}

\section{PREVALENCE OF MALOCLUSIONS IN SCHOOLCHILDREN OF 12 YEARS OLD AT MACHANGARA CUENCA, 2016}

\author{
Aguirre-Suarez Bárbara. ${ }^{1 *}$ \\ ${ }^{1}$ Odontóloga de la República de Chile. Centro médico y dental San José Ltda, Calama.Chile \\ *ba.aguirres@outlook.com
}

\begin{abstract}
Resumen
OBJETIVO. Determinar la prevalencia de maloclusiones en escolares de 12 años de la parroquia Machángara de la ciudad de Cuenca, 2016. MATERIALES Y MÉTODOS. Estudio descriptivo de corte retrospectivo de 184 fichas epidemiológicas de investigación de la Universidad Católica de Cuenca de la evaluación clínica en los planos sagital, transversal y vertical, mediante un muestreo probabilístico aleatorio simple utilizando fórmula para prevalencia, odds ratio y Chi cuadrado. Se analizaron las variables género, gestión escolar y tipo de maloclusión. RESULTADOS. La prevalencia fue de un $81 \%$ de maloclusión, la distribución de maloclusión según género fue de un $83 \%$ en el sexo femenino y $79 \%$ en el sexo masculino; y en relación a la gestión escolar obtuvimos un $81 \%$ de maloclusión en escuelas fiscales y un $75 \%$ en escuelas fiscomisionales. El tipo de maloclusión con mayor frecuencia fue la Clase I con un $38 \%$ seguida de Clase II -1 con un 29\%; Clase III con un $26 \%$ y finalmente la Clase II-2 con un $8 \%$. Las características clínicas observadas con mayor frecuencia fueron $48 \%$ perfil recto, $72 \%$ normodivergencia; $38 \%$ relación molar Clase I; $43 \%$ de relación canina Clase I; $66 \%$ de overjet y overbite normal; $93 \%$ de ausencia de mordida cruzada posterior y $88 \%$ de mordida cruzada anterior; $5 \%$ de mordida abierta; $11 \%$ presencia de diastemas y un $53 \%$ presencia de apiñamiento. CONCLUSIÓN. Existe una alta prevalencia de maloclusiones dentales en escolares de sexo femenino provenientes de escuelas fiscales, de los cuales presentaron una alta frecuencia de características clínicas de maloclusión.
\end{abstract}

Palabras clave: Maloclusión, niño, prevalencia.

\begin{abstract}
OBJECTIVEDetermine the prevalence of malocclusions in 12 years old schoolchildren from the Machangara parish of the city of Cuenca, 2016. MATERIALS AND METHODS. A retrospective cross-sectional study of 184 epidemiological records from the research unit of the Universidad Católica de Cuenca of clinical evaluation at the sagittal, transverse and vertical planes, using a simple random probability sampling using formula for prevalence, odds ratio and Chi square. The variables gender, school management and type of malocclusion were analyzed. RESULTS. The prevalence was $81 \%$ of malocclusion, the distribution of malocclusion according to gender was $83 \%$ female and 79\% male; And in relation to school management we obtained $81 \%$ malocclusion in fiscal schools and $75 \%$ in fiscomision schools. The most frequent type of malocclusion was Class I with $38 \%$ followed by Class II -1 with 29\%, Class III with $26 \%$ and Class II-2 with $8 \%$. The most frequent clinical characteristics were $48 \%$ straight profile; $72 \%$ normodivergence; $38 \%$ Class I molar ratio; $43 \%$ Class I canine relationship; $66 \%$ overjet and normal overbite; $93 \%$ absence of posterior crossbite and $88 \%$ Anterior; $5 \%$ open bite; $11 \%$ presence of diastemas and 53\% presence of crowding. CONCLUSION. There is a high prevalence of dental malocclusions in female schoolchildren from tax schools, of which they presented a high frequency of clinical characteristics of malocclusion.
\end{abstract}

Key words: Malocclusion, child, prevalence.

\section{INTRODUCCIÓN}

La maloclusión dental se define como aquella mal posición dentaria y/o de los huesos maxilares, no solo en sus interacciones fijas sino también dinámicas, en donde las re- laciones con sus antagonistas y con los contactos proximales no son los correctos, produciéndose como consecuencia una desarmonía dentomaxilar. ${ }^{1}$

La clasificación que se aplicó y que se mantiene hasta el día de hoy es la clasificación realizada por el Dr. Ed- 
ward Hartley Angle en 1899, el cual utiliza al primer molar superior por suponer que está en una ubicación esqueletal constante e inalterable en el sistema cráneo facial y propone que las desarmonías dentales eran resultado de alteraciones antero posteriores del maxilar inferior en relación a éste. Es así como Angle estableció las relaciones molares y caninas, clasificándolas en tres clases que se distinguen de la relación oclusal normal denominada clase I molar en donde la cúspide mesio vestibular del primer molar superior permanente ocluye en el surco vestibular del primer molar inferior permanente. ${ }^{1-5}$ La clase II molar es la relación distal en donde la cúspide mesio vestibular del primer molar superior permanente ocluye por delante del surco vestibular del primer molar inferior permanente, dando como consecuencia una retrusión mandibular. Las maloclusiones clase II presentan dos divisiones: división I donde hay protrusión y extrusión de los incisivos superiores y la división II presenta palatinización de los incisivos superiores ${ }^{6-9}$ y por último la clase III molar es la relación mesial en donde el primer molar permanente superior ocluye posterior al surco vestibular del primer molar permanente inferior. La relación canina normal que propone Angle es la clase I de Angle donde la cúspide del canino permanente superior ocluye entre el canino permanente inferior y el primer premolar permanente inferior. ${ }^{10}$ Se refiere a clase II de Angle a la relación canina distal donde la cúspide del canino permanente superior ocluye entre el canino e incisivo lateral inferior permanente; y es clase III de Angle la relación canina mesial donde la cúspide del canino permanente superior ocluye muy distal del canino permanente. $^{11}$

La maloclusión dental no es considerada una entidad patológica, sino es el resultante de alteraciones y/o desórdenes en el sistema estomatognático, es decir, irregularidades en tejidos duros, tejidos blandos y articulación temporomandibular, de carácter multifactorial, determinando así las relaciones dentarias desde el momento del desarrollo prenatal así como postnatal; ${ }^{12}$ por lo tanto las acciones preventivas y de intercepción oportuna, se consideran medidas necesarias en algunos tipos de maloclusiones para disminuir así sus consecuencias y aprovechar la capacidad adaptativa que poseen los componentes del sistema estomatognático. ${ }^{13}$

Según la Organización Mundial de la Salud, las maloclusiones dentales corresponden al $70 \%$ de prevalencia dentro de las alteraciones que afectan a la cavidad bucal, ocupando así el tercer lugar de prevalencia en la clasificación internacional de enfermedades luego de la caries dental y enfermedad periodontal, constituyendo por lo tanto un problema de salud pública. ${ }^{14-17}$

Hoy en día existen pocos estudios que se enfocan en la salud bucal en la comunidad, motivo por el cual se crea la necesidad de efectuar nuestro estudio, con el objetivo principal de determinar y así responder a la pregunta de investigación ¿Cuánto es la prevalencia de maloclusión en los escolares de 12 años de la parroquia Machángara en la ciudad de Cuenca en el año 2016?. El presente estudio está enmarcado en el área de Ortodoncia dentro del Macroproyecto del Mapa Epidemiológico de la salud bucal en la ciudad de Cuenca realizado por el Departamento de Investigación de la Universidad Católica de Cuenca en el 2016.

\section{MATERIALES Y MÉTODOS}

Estudio descriptivo de corte retrospectivo de las fichas epidemiológicas de escolares de 12 años de la parroquia Machángara que cumplían con los criterios de inclusión y exclusión y que están registradas en el programa de libre acceso EPI INFO ver 7.2 en los computadores de la Unidad de Investigación de la Carrera de Odontología en la Universidad Católica de Cuenca.

Los escolares que participaron en el estudio fueron evaluados clínicamente mediante inspección visual por examinadores calibrados por especialistas, los padres firmaron el consentimiento informado y los escolares dieron el asentimiento informado.

El estudio tiene un nivel de originalidad nacional ya que por primera vez se realiza dentro del cantón Cuenca y en la parroquia de Machángara. El proyecto es viable ya que se cuenta con los recursos institucionales, financieros y humanos para la investigación y fue factible ya que se posee el conocimiento de las instituciones escolares, familia y niños involucrados y es un estudio que no implica conflictos bioéticos, debido a que se ejecuta sobre datos ya tomados de pacientes que participaron en el estudio.

Mediante un muestreo probabilístico aleatorio simple se estratificaron proporcionalmente 184 muestras de la población total de 192 fichas de acuerdo a la proporción de escolares de 12 años que tuvo cada centro educativo de la parroquia Machángara del cantón de Cuenca.

En el estudio se analizaron las variables prevalencia de maloclusión dental (perfil anteroposterior, perfil vertical, relación molar, relación canina, overjet, overbite, mordida cruzada anterior y/o posterior, mordida abierta anterior y/o posterior, apiñamiento dentario y diastemas dentarios), género (femenino y masculino) y tipo de gestión escolar (fiscal y fiscocomisional), siendo variables nominales cualitativas.

\section{RESULTADOS}

La muestra estuvo compuesta mayoritariamente por escolares del sexo femenino; ambos sexos provienen mayormente de escuelas fiscales que fiscocomisionales, siendo una muestra no equitativa en ambas variables a estudiar.

La prevalencia de maloclusiones fue de un $81 \%$ en relación a la normo oclusión con un $19 \%$ en la Parroquia Machángara. Gráfico 1. Siendo la Clase I molar la de mayor prevalencia con un $34 \%$ y $40 \%$, seguida de una Clase II división 1 con un $31 \%$ y $27 \%$, una Clase III con un $28 \%$ y $23 \%$ y por último tenemos con una menor prevalencia la Clase II división 2 con un $5 \%$ y $10 \%$ respectivamente en ambos sexos. Gráfico 2. 


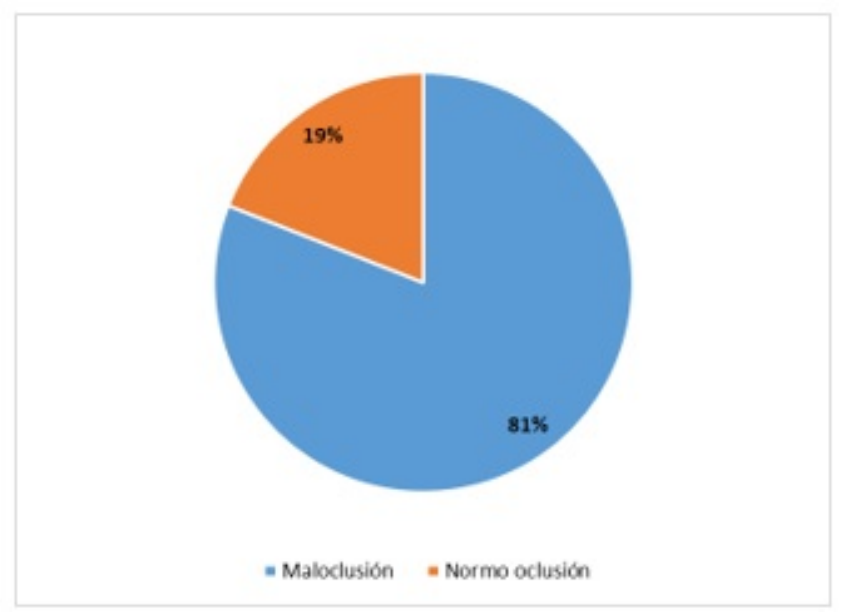

Grf. 1. Prevalencia de maloclusión y normo oclusión de los escolares en la parroquia Machángara.

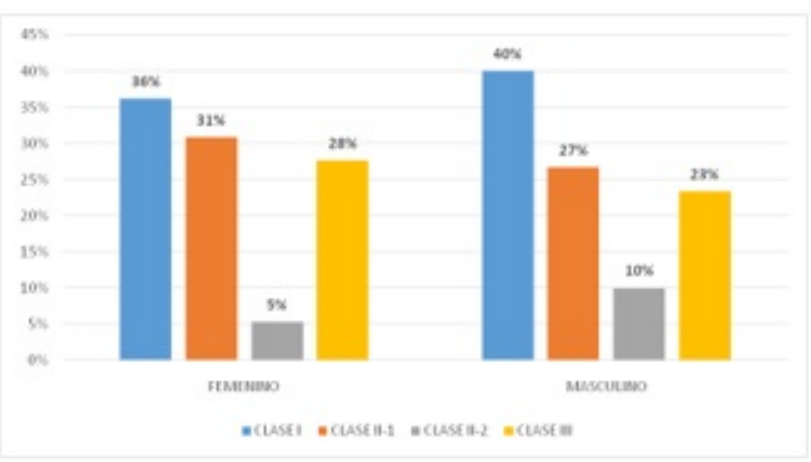

Grf. 2. Distribución de la maloclusión en la Parroquia Machángara.

La maloclusión en relación al género de los escolares se observó que existe una diferencia significativa entre escolares de sexo femenino con un $83 \%$ y el sexo masculino con un $79 \%$. Gráfico 3.

\begin{tabular}{lrr}
\hline \multicolumn{1}{c}{ Examen Extra oral } & $\mathrm{N}^{\circ}$ & \multicolumn{1}{c}{$\%$} \\
\hline Perfil Anteropos terior & & \\
Perfil Cónca vo & 13 & $7 \%$ \\
Perfil Convexo & 82 & $45 \%$ \\
Perfil Recto & 89 & $48 \%$ \\
Perfil Vertical & & \\
Hiperdivergente & 17 & $9 \%$ \\
Hipodivergente & 34 & $18 \%$ \\
Normodivergente & 133 & $72 \%$ \\
& & \\
\hline
\end{tabular}

Tabla 1. Frecuencia de características clínicas extra orales en los escolares de la parroquia Machángara.

En relación a la gestión escolar de las escuelas se observó que existe una diferencia significativa entre maloclusión y normo oclusión en los escolares provenientes de escuelas

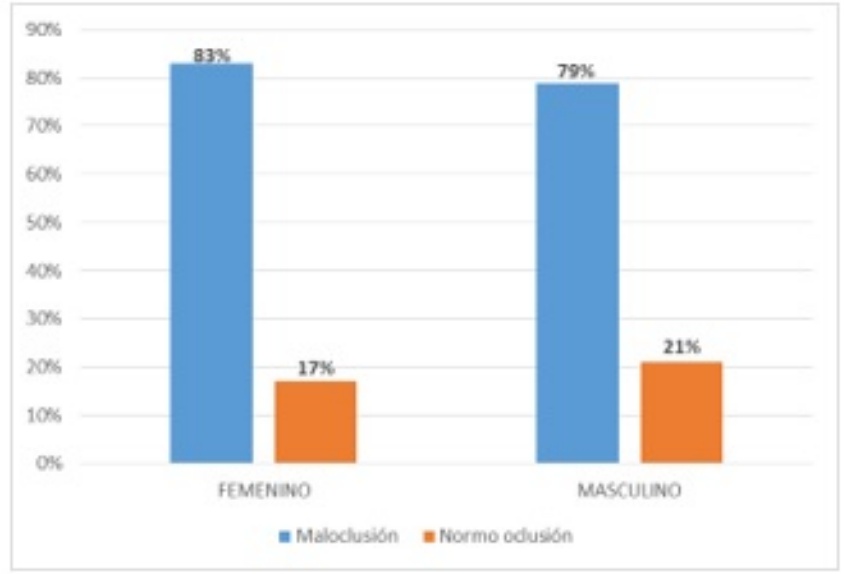

Grf. 3. Maloclusión en relación al género.

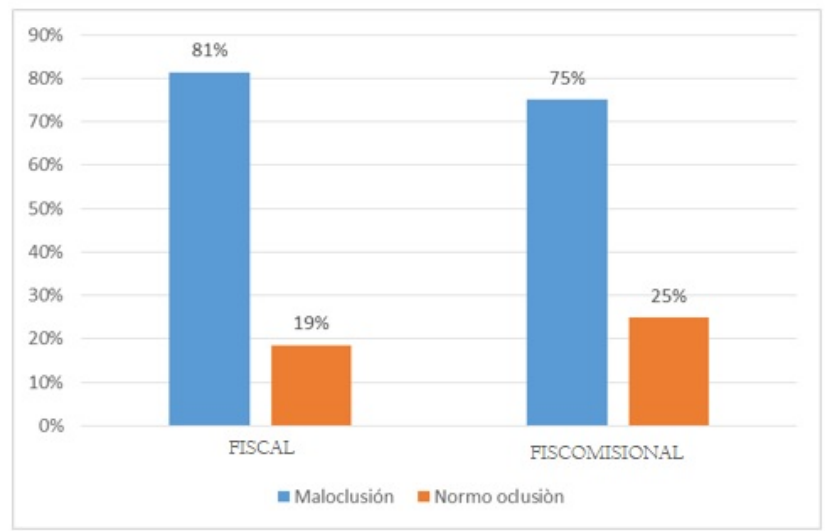

Grf. 4. Maloclusión y normo oclusión en relación a la gestión escolar.

fiscales con un $81 \%$ y escolares provenientes de escuelas fiscocomisionales con un $75 \%$. Gráfico 4 .

En cuanto a las características extraorales se encontró en el perfil anteroposterior, que el perfil recto fue el más predominante con el $48 \%$; en lo que se refiere al perfil vertical, el perfil normodivergente representó el $72 \%$ de la muestra (Tabla 1).

En relación a las características clínicas intraorales se observó con mayor frecuencia: clase I molar derecha (58\%) e izquierda (54\%); Clase I canina derecha e izquierda con un $43 \%$; overjet y overbite con valores normales con un $66 \%$; ausencia de mordida abierta anterior o posterior (95\%); ausencia de mordida cruzada anterior $(88 \%)$ y posterior $(93 \%)$; ausencia de diastemas dentarios con un $89 \%$ y presencia de apiñamiento dentario con un $53 \%$ distribuido entre leve, moderado y severo. (Tabla 2). 


\begin{tabular}{|c|c|c|}
\hline Examen Intra oral & $\mathrm{N}^{\circ}$ & $\%$ \\
\hline \multicolumn{3}{|l|}{ Llave Molar } \\
\hline Clase I molar derecha & 108 & $58 \%$ \\
\hline Clase II molar derecha & 29 & $16 \%$ \\
\hline Clase III molar dere cha & 44 & $24 \%$ \\
\hline N.A. & 5 & $3 \%$ \\
\hline Clase I molar izquierda & 99 & $54 \%$ \\
\hline Clase II molar izquierda & 37 & $20 \%$ \\
\hline Clase III molar izquierda & 44 & $24 \%$ \\
\hline N.A. & 4 & $2 \%$ \\
\hline \multicolumn{3}{|l|}{ Llave Canina } \\
\hline Clase I can ina dere cha & 79 & $43 \%$ \\
\hline Clase II canina derecha & 44 & $24 \%$ \\
\hline Clase III canina derecha & 27 & $15 \%$ \\
\hline N.A. & 34 & $18 \%$ \\
\hline Clase I canina izquierda & 79 & $43 \%$ \\
\hline Clase II canina zquierda & 38 & $21 \%$ \\
\hline Clase III canina izquierda & 27 & $15 \%$ \\
\hline N.A. & 40 & $22 \%$ \\
\hline \multicolumn{3}{|l|}{ Sentido Sagital: Overjet } \\
\hline O verjet ausente $(-1 \mathrm{~mm})$ & 21 & $11 \%$ \\
\hline Overjet normal (1-3 mm) & 121 & $66 \%$ \\
\hline O ve rjet a umentado $(+3 \mathrm{~mm})$ & 42 & $23 \%$ \\
\hline \multicolumn{3}{|l|}{ Sentido Vertical: Overbite } \\
\hline Overbite ausente $(-1 \mathrm{~mm})$ & 22 & $12 \%$ \\
\hline Overbite normal ( $1-3 \mathrm{~mm})$ & 121 & $66 \%$ \\
\hline Overbite aumentado $(+3 \mathrm{~mm})$ & 41 & $22 \%$ \\
\hline \multicolumn{3}{|l|}{$\begin{array}{l}\text { Sentido Vertic al: Mordida } \\
\text { A bierta }\end{array}$} \\
\hline Mordida Anterior ausente & 174 & $95 \%$ \\
\hline Mordida Anterior presente & 10 & $5 \%$ \\
\hline \multicolumn{3}{|l|}{ Sentido Transversal } \\
\hline $\begin{array}{l}\text { Mordida Cruzada Anterior } \\
\text { ausente }\end{array}$ & 161 & $88 \%$ \\
\hline Mordida Cruzada Anterior & 18 & $10 \%$ \\
\hline Localizada & & \\
\hline $\begin{array}{l}\text { Mordida Cruzada Anterior } \\
\text { Generalizada }\end{array}$ & 5 & $3 \%$ \\
\hline Mordida Cruzada Posterior & 171 & $93 \%$ \\
\hline \multicolumn{3}{|l|}{ Ausente } \\
\hline $\begin{array}{l}\text { Mordida Cruzada Posterior } \\
\text { Unilateral }\end{array}$ & 8 & $4 \%$ \\
\hline $\begin{array}{l}\text { Mordida Cruzada Posterior } \\
\text { Bilateral }\end{array}$ & 5 & $3 \%$ \\
\hline \multicolumn{3}{|l|}{ Maloclus iones Inter arco } \\
\hline Diastemas ausencia & 164 & $89 \%$ \\
\hline Diastemas leves & 16 & $9 \%$ \\
\hline Diastemas moderados & 4 & $2 \%$ \\
\hline A piñam ento ausencia & 87 & $47 \%$ \\
\hline A piñamento leve & 47 & $26 \%$ \\
\hline A piñamento moderado & 40 & $22 \%$ \\
\hline A piñamento severo & 10 & $5 \%$ \\
\hline
\end{tabular}

Tabla 2. Frecuencia de características clínicas intra orales en los escolares de la parroquia Machángara.

\section{DISCUSIÓN}

El presente estudio se enfocó principalmente en determinar la prevalencia de maloclusiones en los escolares de 12 años, tuvo un resultado de un $81 \%$ de prevalencia, distribuido en un $83 \%$ en el sexo femenino y un $81 \%$ de escolares provenientes de escuelas fiscales, estos resultados no son solo observados a nivel regional en Ecuador sino que se observa a nivel latinoamericano y mundial.

Valdés y cols ${ }^{18}$ en el estudio realizado para determinar prevalencia de maloclusiones, de los 140 niños evaluados un $89,3 \%$ presentaron maloclusiones dentales.

En relación a la prevalencia según el género de los escolares Ramos y cols nos muestra que del $60,8 \%$ de escolares que presentaron maloclusión, el 57,5\% corresponde a escolares de sexo femenino, así mismo en los estudios de Plazas y cols, ${ }^{19}$ y el de Palacios y cols, ${ }^{20}$ nos muestran una mayor prevalencia de maloclusiones en escolares del sexo femenino con un $52 \%$.

En relación a la gestión escolar, el estudio de López ${ }^{21}$ con una muestra de 143 escolares, el $83 \%$ de niños con maloclusión dental proviene de escuelas fiscales.

El estudio de Burgos, ${ }^{22} 2014$ realizado en Chile nos muestra que existe una mayor prevalencia de Clase I $(51,1 \%)$, el estudio de Aliaga y cols ${ }^{23}$ realizada en la Amazonia de Ucayali, Perú mostró una prevalencia de Clase I $(58 \%)$.

En relación a la frecuencia de las características clínicas el estudio de Plazas y cols, ${ }^{24}$ mostró una mayor prevalencia del perfil convexo (58,3\%); en el estudio de Palacios y cols, ${ }^{25}$ se observó un alto porcentaje de relación canina Clase I derecha (55\%) e izquierda (58\%); el estudio de Urrego y cols ${ }^{21}$ presentó un $68,6 \%$ de overjet normal de $1-3 \mathrm{~mm}$; en el estudio de Urrego y cols, ${ }^{21}$ el $48,6 \%$ presentó overbite normal de 1-3 mm; en el estudio de Burgos solo el 3,8\% presentó mordida abierta al igual que el estudio de Chumi y et al., ${ }^{16}$ el $5 \%$ presentó mordida abierta anterior; en el estudio de Urrego y cols, ${ }^{21}$ se presentó un $11 \%$ de mordida cruzada anterior o posterior de los cuales $10,6 \%$ es uni lateral y un $3.7 \%$ es bilateral; en el estudio de Mendoza y cols, ${ }^{20}$ se muestra un $46 \%$ de apiñamiento y en relación a los diastemas dentarios el estudio de Urrego y cols, ${ }^{21}$ nos muestra solo un $11,3 \%$ de diastemas.

\section{CONCLUSIÓN}

El presente estudio de investigación nos arrojó una elevada prevalencia de maloclusiones dentales con un $81 \%$ en los escolares de la parroquia Machángara, siendo mayor en el sexo femenino en relación al masculino y en escuelas fiscales en relación a las fiscocomisionales. Estos porcentajes son motivo para implementar medidas de promoción, prevención y de intercepción oportuna enfocada en la parroquia y en el cantón Cuenca para disminuir estos porcentajes a futuro.

Conflicto de intereses y financiamiento Los autores declaran no tener conflicto de intereses, haber cumplido con los requisitos de autoría y haber autofinanciado este artículo.

Agradecimiento: Al Dr Ebingen Villavicencio coordinador del departamento de investigación. 


\section{Referencias}

1 Ministerio de Salud Pública del Ecuador. Protocolos Odontológicos. Ed. Dirección Nacional de Normalización. 1ed.

2 Alemán M, Martínez I, Pérez A. Necesidad de Tratamiento ortodóncico en escolares. Aplicación del índice DAI. Rev Méd Electrón [Internet]. 2011 Jun-Jul [citado: fecha de acceso]; 33(4). Documento disponible.

3 Solarte J, Rocha A, Agudelo A. Perfil epidemiológico de las alteraciones de la oclusión en la población escolar del corregimiento de Genoy, municipio de Pasto, Colombia. Rev Fac Odontol Univ Antioq [Internet]. 2011; 23(1): 111125. Documento disponible.

4 Burgos D. Prevalencia de maloclusiones en niños y adolescentes de 6 a 15 años en Frutillar, Chile. Int. J. Odontostomat [Internet]. 8(1):13-19, 2014. Documento disponible.

5 González R, Ochoa D, Silva C, Cruz I. Anomalías dentofaciales y hábitos deformantes en alumnos de una escuela primaria. Rev haban cienc méd [Internet]. 2012 Dic [citado 2016 Ago 01]; 11( 4 ): 464-473. Documento disponible.

6 Podadera Z, Rezk A, Flores L, Ramírez M. Caracterización de las anomalías dentomaxilofaciales en niños de 6 a 12 años. Rev Ciencias Médicas [Internet]. 2013 Oct [citado 2016 Ago 01]; 17(5): 97-108. Documento disponible.

7 Díaz H, Ochoa B, Paz L, Casanova K, Coca Y. Prevalencia de maloclusiones en niños de la escuela Carlos Cuquejo del municipio Puerto Padre, Las Tunas. Medisur [Internet]. 2015 Ago [citado 2016 Ago 01] ; 13(4):494-499. Documento disponible.

8 Proffit W. Ortodoncia contemporánea: teoría y práctica. 3ra. Ed. Madrid. Ed. Elsevier. 2001.

9 Vellini F. Ortodoncia. Diagnóstico y planificación clínica. Editorial Amolca. Madrid. 2002.

10 Medrano I. Curso de tratamiento "Introducción a la prostodoncia y oclusión". Pagina web. Slideplayer. 2014. Documento disponible.

11 Colina R, Barbosa M. Tipos de mordida. [Internet]. Documento disponible.

12 Calderón P. Cirugía oral y Maxilofacial. Análisis de la oclusión. Página web. 2013. Documento disponible.

13 Gómez E. Ortodoncia al día. Casos clínicos. Página web. 2011. Documento disponible.

14 Pan American Health Organization. Health in the Americas. Washington DC: PAHO; 1998. P. 413-27.

15 Castañeda M. Historia Clínica en Ortodoncia. Slideplayer. 2015. Documento disponible.

16 Chumi R, Cordero M. Prevalencia de anomalías Dentomaxilares en adolescentes del Colegio"Miguel Cordero Crespo"de 12 a 14 años, en el Cantón Cuenca-Ecuador, año 2014. Rev. Latinoamericana de Ortodoncia y Ortopedia [Internet]. 2014. Documento disponible.

17 Palacios L, Carrillo D. Prevalencia de maloclusiones de Angle en niños de 9 a 13 años. USFQ. Revista Odonto investigación [Internet]. 2015. Documento disponible.
18 Aliaga A, Mattos M, Aliaga R, Del Castillo C. Maloclusiones en niños y adolescentes de caseríos y comunidades nativas en la Amazonía de Ucayalí, Perú. Rev Perú MedExp Salud Pública [Internet]. 2011; 28(1): 87-91. Documento disponible.

19 Plazas J, Martínez O, Castro L, Solana A, Villalba L. Prevalencia de maloclusiones en niños de una escuela en Cartagena de Indias, Colombia año 2011. Rev Cien Salud 3(1), 2-8 [Internet]. Documento disponible.

20 Mendoza L, Meléndez A, Ortiz R, Fernández A. Prevalencia de las maloclusiones asociada con hábitos bucales nocivos en una muestra de mexicanos, año 2014. Revista Mexicana de ortodoncia, 2(4), 220-227. [Internet]. Documento disponible.

21 Urrego P, Jiménez L, Londoño M, Zapata M, Botero P. Perfil epidemiológico de la oclusión dental en escolares de Envigado, Colombia año 2011. Revista de Salud Pública, 13(6), 1010-1021. Documento disponible.

22 Bermúdez P, Arbeláez A, Guerra J, Correa D, Fernández L, Moncada N, Usuga C. Perfil epidemiológico de la oclusión dental, en escolares de 6 a 12 años, del colegio universidad cooperativa de Colombia, corregimiento de San Antonio de prado, 2010. Revista Colombiana de Investigación en Odontología, 2(5), 134-140. Documento disponible.

23 López E. Estudio de prevalencia de mal oclusión en dentición en niños de 6 a 12 años de escuelas de la ciudad de Quito, provincia de Pichincha, Ecuador, en el período 2015. Facultad de Odontología. UDLA. Quito. 68 p. Documento disponible.

24 Villavicencio E, Cuenca K, Vélez E, Sayago J, Cabrera A. Pasos para la planificación de un estudio clínico, 2016. Odontología activa. Universidad Católica de Cuenca ISSN: 2477-8915. Documento disponible.

25 Villavicencio E. El tamaño muestral en tesis de postgrado. ¿Cuántas personas debo encuestar?, 2011. Documento disponible.

Recibido: 13 de Septiembre de 2017.

Aceptado: 17 de Diciembre de 2017. 
